\title{
A Compact Tapered-slot-feed Annular Slot Antenna for Ultra-wideband Applications $\dagger$
}

\author{
Tzyh-Ghuang $\mathrm{Ma}^{*}$ and Shyh-Kang Jeng \\ Graduate Institute of Communication Engineering and \\ Department of Electrical Engineering, \\ National Taiwan University, Taipei, Taiwan, R.O.C. \\ Email: skicng@iew.ec.ntu.edu.tw.
}

A novel compact tapered-slot-fed annular slot antenna suitable for ultra-wideband (UWB) applications is presented. Frequency domain as well as time domain characteristics of the antenna are well investigated and discussed. A measure to quantify the performance of the radiation patterns of such an ultra-wideband antenna is described.

\section{Introduction}

Ultra-wideband (UWB) technologies have experienced a blooming growth since the FCC in the States issued the regulations for unlicensed applications. Studies in various fields including hardwarc implementation [1], pulse shape optimization [2] and antenna designs [3]-[5] etc. have been carried out to demonstrate the promising future of this technology. Differ from the conventional narrowband communication systems, the UWB requires bandwidth of several $\mathrm{GHz}$ and transmits nanosecond pulses without any carrier. The antenna implemented in the UWB system plays an important role than it in any other system since it actually behaves like a bandpass filter to reshape the spectra of the pulses and hence need to be designed carefully to avoid unwanted distortions. Literatures discussing the antennas suitable for UWB applications have been reported [3][4]. Generally speaking, it is quite challenging to design a proper antenna to satisfy the critical requirements of such systems including ultra-wide bandwidth, omnidirectional patterns, constant gain and linear phase over the entire band, high radiation efficiency, low profile and easy to manufacture. Recently, we introduced a novel planar antenna that can be easily manufactured with photolithography techniques [5][6]. It consists of a novel ultra-wideband taper slot feed and dipole-type radiating elements and proven to be adequate for UWB applications. In this paper, a new tapered-slot-feed annular slot antenna with much more compact size is proposed. The performance of the rovel antenna is comparable to that of [5]. The geometry of the antenna is described in Section II. The frequency domain as well as time domain characteristics of the antenna are illustrated in Sections III and IV, respectively. Some conclusions are drawn in Section V.

\section{Antenna Geometry}

The geometry of the proposed antenna is shown in Fig. 1. The inner boundary of the annular slot is described by the elliptical tapered profile of the feeding structure and a semicircle centered at $C_{n}$, whereas the outer boundary of that is depicted with a concentric arc which intersected the centerline of the antenna at $\mathbf{A}$. The slot width is kept as $10 \mathrm{~mm}$. The dimensions of the microstrip line-to-slotline transition are optimized to maximize the antenna bandwidth. The overall size of the antenna is about $4^{*} 6 \mathrm{~cm}^{2}$, which is almost half of that in [5] with comparable operating bandwidth. The proposed antenna is simulated using Ansoft HFSS ${ }^{\phi}$ 8.0 and fabricated on Rogers RT/Duroid 5880 substrate with thickness of 62 mil and dielectric constant of 2.2. The simulated and measured return losses are illustrated in Fig. 2, which covers the whole UWB band from 3.1 to $10.6 \mathrm{GHz}$. The little discrepancy between them can be attributed to the manufacture error as well as the inaccuracy of the antenna model implemented in the simulation.

$\dagger$ This work is supported by the National Science Council, Republic of China, under Grant of NSC 92-2213-E002-067. 


\section{Frequency Domain Characteristics}

Typical radiation patterns in both $H$ and $E$-planes at $6.5 \mathrm{GHz}$ are shown in Figs. 3(a) and (b), respectively. The dominant field component of the proposed antenna is the $E_{\theta}$ component. As shown in the figures, the $H$-plane (xy-plane) pattern is rather uniform whereas the $E$-plane ( yz-plane) pattern exhibits dual-polarized properties. Moreover, the field pattern of the dominant field component, $E_{g}$, of the antenna demonstrates a donut-like profile, which is analogous to that of a half-wavelength dipole, up to $8.5 \mathrm{GHz}$.

The antenna transfer function is another issue of concern, from which we could realize how the spectra of the pulses might be modified upon the receiving antenna. For UWB applications, the magnitude of the antenna transfer functions should keep constant in the operating band but drop rapidly outside the band to satisfy the emission mask issued by FCC. In addition, the phase response of the transfer function needs to be linear over the entire band. In this work, the antenna transfer functions are evaluated using

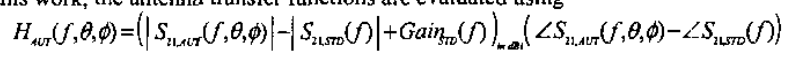

where $S_{11, u r t}(f, \theta, \phi)$ is the measured frequency response of the antenna under test at angle $(\theta, \phi)$ and $S_{1, s s p}(f)$ is that of a standard antenna with specific gain, $\operatorname{Gain}_{\text {sso }}(f)$. Figure 4(a) illustrates the magnitude of the measured antenna transfer functions in the $H$-plane at $\phi=0^{\circ}, 90^{\circ}, 180^{\circ}, 270^{\circ}$. From the figure, we observe that the measured tran sfer functions of the proposed antenna are rather flat throughout the band except in the backward direction. The corresponding antenna gain remains almost unchanged and is about $5 \mathrm{dBi}$ up to $9 \mathrm{GHz}$. In addition, the phase response of the antenna transfer function in the $H$-plane at $\phi=0^{\circ}$ is depicted in Fig. 4(b) and linear phase response is demonstrated. Consequently, the proposed antenna is proven to be applicable for UWB applications. Note that all the measurements are performed in an anechoic chamber from 1 to $18 \mathrm{GHz}$ and the standard antenna is a double ridged horn antenna manufactured by Spectnum Technologies International with model no. DRH-118.

\section{Time Domain Responses}

Time domain responses of the proposed antenna are evaluated using the antenna transfer functions obtained in the previous section. The source pulse at the excitation is assumed to be the first derivative of the Gaussian function and the transmitting system is taken as an ideal differentiator, which in tum implies the input pulse at the receiving antenna terminal will be the second derivative of the Gaussian function,

$$
\operatorname{Input}(t)=A\left(1-4 \pi\left(t / T_{-}\right)^{2}\right)\left(\exp \left(-2 \pi\left(t / T_{-}\right)^{2}\right)\right)
$$

The spectrum of the input pulse is then multiplied with the measured antenna transfer function and an inverse Fourier transform is performed to achieve the required time domain response. This can be expressed by$$
\operatorname{Outpur}(t, \theta, \phi)=\Im^{-1}\left\{\operatorname{Input}(f) \cdot H_{\text {ttr }}(f, \theta, \phi) \cdot \Pi(f)\right\}
$$

where $\Pi(f)$ represents an ideal bandpass filter from 1 to $18 \mathrm{GHz}$. The output waveforms at the receiving antenna terminal in the $H$-plane at $\phi=0^{\circ}, 90^{\circ}, 180^{\circ}, 270^{\circ}$ are depicted in Figs. $5(a)$ and (b). Range related effects are calibrated from the antenna transfer functions so that no propagation delay is shown here. Well-behaved time domain responses are proved and the tate time ringing is almost negligible. Again, it demonstrates the applicability of the proposed antenna for UWB applications. More discussions upon the time domain responses will be given during the presentation.

\section{v. Conclusions}

A novel tapered-slot-fed annular stot antenna has been proposed and proved to be suitable 
for ultra-wideband applications. The frequency domain as well as time domain characteristics of the antenna are well investigated and discussed. The antenna is much more compact than those in [5] and thus can be easily integrated with other components. In addition, the definition of the uniformity is given and helpful in qualifying the performance of the radiation patterns of such an ultra-wideband antenna.

\section{References}

[1] G. Ossberger, T. Buchegger, A. Stelzer, A. Springer, C. Diskus, and R. Weigel, "Ultra wideband test set-up with a novel low-cost pulse forming network," in Proc. Asia-Pacific Microwave Conference, vol. 3, Seoul, Korea, Nov. 2003 pp. 2056-2059.

[2] B. Parr, B. Cho, K. Wallace and Z. Ding, "A novel ultra-wideband pulse design algorithm," IEEE Commun. Lett., vol. 7, pp.219-221, May 2003.

[3] Z. N. Chen, X. H. Wu, N. Yang and M. Y. W. Chia, "Design consideration for antennas in UWB wireless communication systems," in IEEE AP-S Int. Symp. Dig., vol. 1, Columbus, OH, June 2003, pp. 822-825.

[4] X. Qing, M. Y. W. Chia and X. Wu, "Wide-slot antenna for UWB applications," in IEEE AP-S Int. Symp. Dig, vol. 1, Columbus, OH, June 2003, pp. 834-837.

[5] T. G. Ma and S. K. Jeng, "A novel compact ultra-wideband printed dipole antenna with tapered slot feed," in IEEE AP-S Int. Symp. Dig., vol. 3, Columbus, $\mathrm{OH}$, June 2003, pp. 608-611.

[6] T. G. Ma and S. K. Jeng, "A parameter study of a novel ultra-wideband printed dipole antenna with tapered slot feed," in Proc. Asia-Pacific Microwave Conference, vol. 3, Seoul, Korea, Nov. 2003,pp. 1981-1984

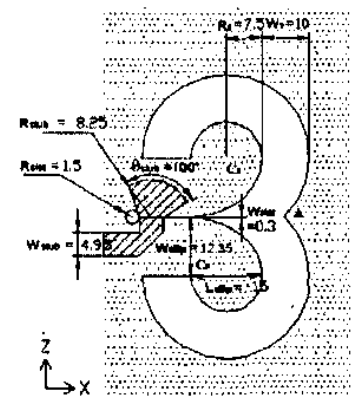

Fig. 1 Geometry of the antenna

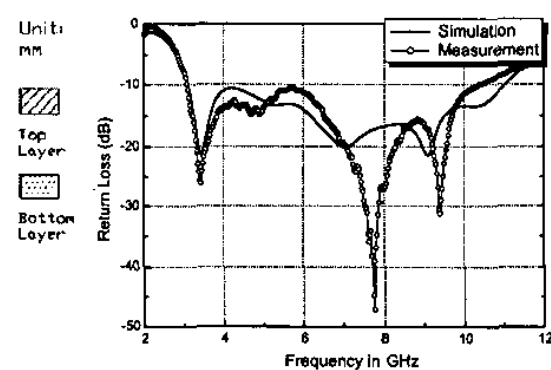

Fig. 2 Simulated and measured retum losses of the antenna. 


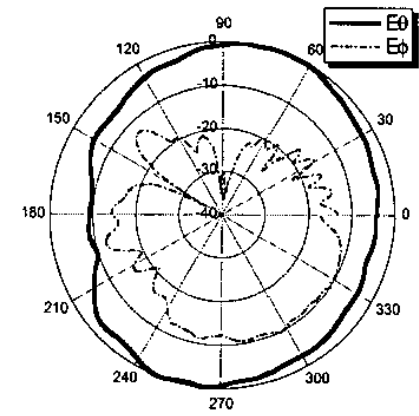

(a)

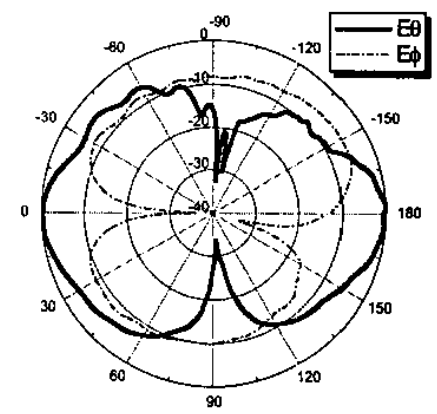

(b)

Fig. 3 Measured (a) $H$-plane and (b) $E$-plane radiation patterns at $6.5 \mathrm{GHz}$.

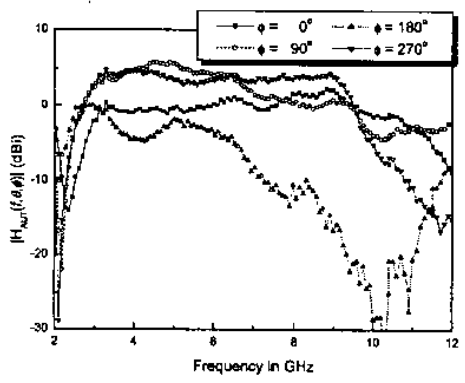

Fig. 4(a) Magnitude of the measured antenna transfer functions in the $H$-plane at $\phi=0^{\circ}, 90^{\circ}, 180^{\circ}, 270^{\circ}$.

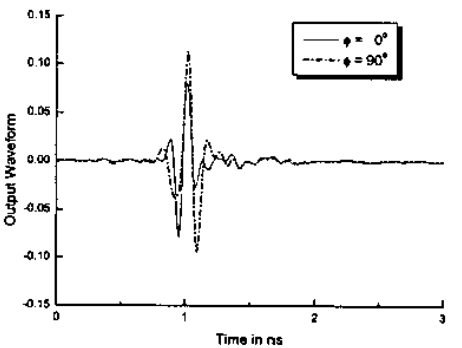

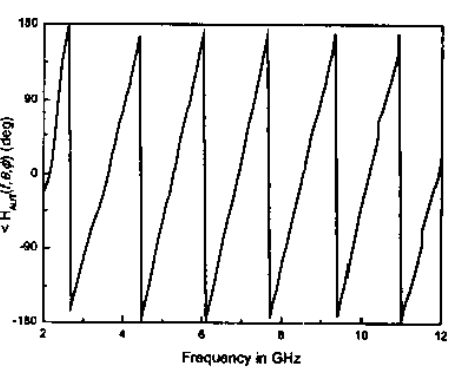

Fig. 4(b) Phase response of the measured antenna transfer function in the $H$-plane at $\phi=0^{\circ}$.

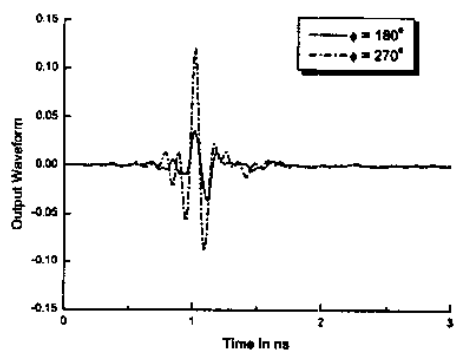

Fig. 5 Output waveforms at the receiving antenna terminal in the $H$-plane at (a) $\phi=0^{\circ}, 90^{\circ}$ and (b) $\phi=180^{\circ}, 270^{\circ}$. 